\title{
Optimization of mesoporous titanosilicate catalysts for cyclohexene epoxidation via statistically guided synthesis
}

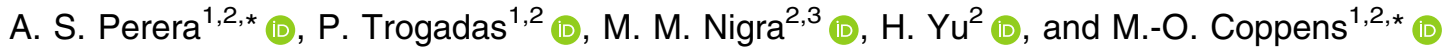 \\ ${ }^{1}$ Centre for Nature Inspired Engineering, University College London, Torrington Place, London WC1E 7JE, UK \\ ${ }^{2}$ Department of Chemical Engineering, University College London, Torrington Place, London WC1E 7JE, UK \\ ${ }^{3}$ Department of Chemical Engineering, University of Utah, Salt Lake City, UT 84112, USA
}

Received: 16 November 2017

Accepted: 18 January 2018

Published online:

29 January 2018

(C) The Author(s) 2018. This article is an open access publication

\begin{abstract}
An efficient approach to improve the catalytic activity of titanosilicates is introduced. The Doehlert matrix (DM) statistical model was utilized to probe the synthetic parameters of mesoporous titanosilicate microspheres (MTSM), in order to increase their catalytic activity with a minimal number of experiments. Synthesis optimization was carried out by varying two parameters simultaneously: homogenizing temperature and surfactant weight. Thirteen different MTSM samples were synthesized in two sequential 'matrices' according to Doehlert conditions and were used to catalyse the epoxidation of cyclohexene with tert-butyl hydroperoxide. The samples (and the corresponding synthesis conditions) with superior catalytic activity in terms of product yield and selectivity were identified. In addition, this approach revealed the limiting values of each synthesis parameter, beyond which the material becomes catalytically ineffective. This study demonstrates that the DM approach can be broadly used as a powerful and time-efficient tool for investigating the optimal synthesis conditions of heterogeneous catalysts.
\end{abstract}

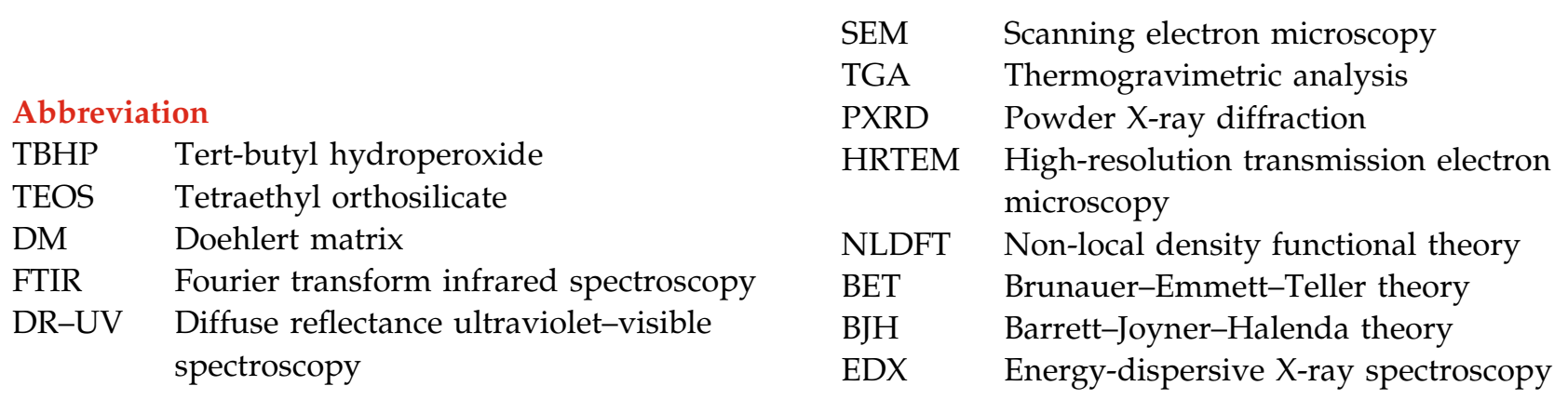

Address correspondence to E-mails: ayomi.perera@ucl.ac.uk; m.coppens@ucl.ac.uk 


\section{XPS X-ray photoelectron spectroscopy \\ $\mathrm{H}_{2} \mathrm{O}_{2} \quad$ Hydrogen peroxide \\ ICP-AES Inductively coupled plasma-atomic emission spectroscopy}

\section{Introduction}

Synthetic porous titanosilicates are widely applied as active and selective heterogeneous catalysts to produce industrially relevant organic precursors [1-5]. Since their introduction in 1983, various categories, such as microporous [6-8], mesoporous [9-13] and layered types [14-19], have been synthesized. These target different applications, such as ion exchange [20-24], adsorption [25-28], membrane separation [29-32], pillaring templating [33,34] and oxidation catalysis [35-38].

Despite the diversity and versatility of titanosilicates, there remain challenges in synthesizing the preferred structures. It is desirable to optimize synthesis procedures to facilitate materials development in a faster, more reproducible way [39]. The requirement of specialized conditions [40, 41] and high sensitivity to changes in synthesis conditions often results in lower than expected activity of catalyst candidates [42-44]. Hence, the use of a statistically guided approach could greatly benefit the optimization of titanosilicate synthesis. However, conventional optimization techniques, such as factorial, Plackett-Burman, central composite, Box-Behnken and orthogonal designs, have many disadvantages [45-47].

Factorial designs or Plackett-Burman designs, for example, are first-order models or univariate systems, where only one parameter can be studied at the same time for optimization [46]. Full factorial models can be used to investigate every factor at two levels. For a large number of factors, however, a fractional factorial model becomes necessary. The limitation of allowing only two factors to be investigated at any one time is disadvantageous, as many processes are complex, requiring multivariate systems in order to simultaneously and properly study the effects of several parameters. In such instances, second-order models, which involve more than two factors, such as central composite design (CCD), Box-Behnken design (BBD), Doehlert matrix design or orthogonal design (OD) can be used.
CCD has some advantages, as it requires fewer experiments than the full factorial, three-level designs [48]. It combines full or fractional factorial designs with additional star points and a centre point, and can fit quadratic polynomials, with rotatable or orthogonal properties. BBD is another second-order, rotatable model, that allows the proportional increase in the number of design points, to the number of polynomial coefficients, and is based on an incomplete factorial design with three levels [49].

OD is another well-known type of factorial design [50]. By definition, factor analysis becomes 'orthogo-

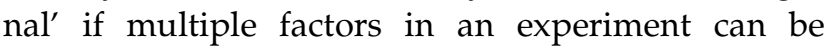
analysed independently of each other. OD is very useful to analyse a small but representative sample from a large set of data, when analysing all data sets is not practical [51]. However, this technique is not always useful, particularly in material synthesis, when multiple experimental parameters have a combined significant effect on the final product formed, because they depend on each other.

The Doehlert matrix (DM) is also a second-order model, but has distinct advantages over factorial, CCD, BBD and OD models [45, 47, 52]. Doehlert designs are simpler and allow optimization of a process or reaction with fewer experiments, thus saving time and cost. They can be moved through the experimental space effectively, and results from previous experiments can be used to guide subsequent, adjacent experimental points (ESI, Figure S1) [53]. Also, they allow the user to adjust the range of each parameter, at each level of the related factors, especially if it is difficult to obtain a definite optimum point.

The DM approach becomes particularly advantageous, when the simultaneous effects of multiple experimental parameters become significant for optimizing a process. Synthesis conditions are often affected by fractions of parameter values; thus, the latter may need to be probed based on a range that contains fractions of the minimum and maximum of the range. The DM gives the investigator this freedom. In addition, the DM approach allows sequential investigation of regions in matrix space, based on previously obtained results. Thus, if a researcher recognizes a region of favourable reaction parameters, but not necessarily one optimal point, another matrix can be built around or in the direction of interest. Moreover, the range of experimental parameters can be narrowed down, if necessary, to 
enhance the focus around a region of interest (ESI, Figure S1). Hence, the DM approach has been widely utilized in analytical sciences, as an optimization tool and as a simple alternative to orthogonal design [47]. The method has been utilized successfully in oil refining, homogeneous catalysis, heavy metal analysis and food science, for optimization of experimental and analytical parameters [54-59]. ESI, Sects. 1 and 2, contains a more detailed discussion of the Doehlert matrix approach.

Herein, we introduce the use of the DM model as an efficient, versatile statistical optimization method, to investigate optimal synthesis conditions of titanosilicates. We demonstrate that this model is particularly desirable for a complex synthesis problem, such as the development of titanosilicates for epoxidation catalysis. Traditionally, the synthesis of titanosilicates has been carried out via techniques such as direct hydrothermal synthesis [60-62], the dry gel conversion method [63] and the post-synthesis method [64]. Later on, amorphous varieties became prominent, targeting bulky organic substrates and oxidants $[39,65]$, as opposed to the conventional, ordered, crystalline [66-68] types, which were suitable for smaller reactants. Soft templating techniques have been utilized to generate such amorphous titanosilicates along with uniform particle shape and size, and increased surface area and robustness [44, 45]. However, statistically guided optimization of the synthesis of titanosilicates is not widely reported.

The goal of this study was to develop a systematic approach to improve the catalytic activity of titanosilicates, via optimization of the synthesis conditions, utilizing the Doehlert matrix model. A mesoporous titanosilicate (MTSM) was synthesized, via surfactant templating, as an effective heterogeneous catalyst for the epoxidation of cyclohexene with TBHP as oxidant. The effect of the pore structure and the amount of framework $\mathrm{Ti}^{4+}$ on catalytic performance was also investigated.

\section{Experimental}

\section{Synthesis of MTSM material}

Synthesis was based on procedures that were modified from those published by Li and Coppens [39]. All chemicals were purchased from Sigma-Aldrich and used without further purification.

\section{General procedure}

$1.0 \mathrm{ml}$ of $\mathrm{Ti}(\mathrm{IV}) n$-butoxide (99\%) was added dropwise to $30.0 \mathrm{ml}$ of DI water $(18.2 \mathrm{M} \Omega)$, at $4{ }^{\circ} \mathrm{C}$, under magnetic stirring, in order to form $\mathrm{Ti}(\mathrm{OH})_{4}$ precipitate. The precipitate was filtered under vacuum and washed with DI water. The $\mathrm{Ti}(\mathrm{OH})_{4}$ was then dissolved in $4.0 \mathrm{ml}$ of $4 \mathrm{~N} \mathrm{HNO}_{3}$ to produce $\mathrm{TiO}\left(\mathrm{NO}_{3}\right)_{2}$ active species. The $\mathrm{TiO}\left(\mathrm{NO}_{3}\right)_{2}$ was then mixed with a solution of $6.6 \mathrm{ml}$ tetraethyl orthosilicate (TEOS, 98\%) and $2.0 \mathrm{ml}$ ethanol and stirred vigorously for $30 \mathrm{~min}$, to form isolated $\mathrm{Ti}^{4+}$ sites in the silica matrix. The physical structure of this liquid mixture was then transformed into mesoporous microspheres by surfactant templating. The titanosilicate mixture was added to a mixture of $26.1 \mathrm{~g}$ kerosene and $7.9 \mathrm{~g}$ Span 80 , and homogenized with an Ultra-Turrax homogenizer, at $3000 \mathrm{rpm}$ for $2 \mathrm{~h}$, at $80^{\circ} \mathrm{C}$. The microspheres formed were then vacuum-filtered and washed with acetone and DI water, followed by drying at $80^{\circ} \mathrm{C}$ for $2 \mathrm{~h}$. Finally, materials were calcined at $750{ }^{\circ} \mathrm{C}$ for $6 \mathrm{~h}$.

\section{Characterization}

FTIR spectroscopy was carried out with a Bruker Vertex 70 instrument. Raman spectroscopy was conducted with a Renishaw InVia Raman microscope using a Kimmon $\mathrm{He} / \mathrm{Cd}$ laser at $442 \mathrm{~nm}$ wavelength. SEM images were taken using JEOL JSM-6480LV and JEOL JSM-5410LV scanning electron microscopes. EDX was performed using the above instruments in low vacuum mode. HRTEM images were taken with a JEOL 2100 microscope operating at $200 \mathrm{keV}$. The samples were dispersed in methanol and then dried on a Holey carbon film $\mathrm{Cu}$ grid, for TEM observation. Thermogravimetric analysis (TGA) was performed on a PerkinElmer TGA7, at 303-1273 K using dry air flow with a heating rate of $10 \mathrm{~K} / \mathrm{min}$. Powder X-ray diffraction (PXRD) patterns were obtained with a Stoe STADI-P instrument, using $\mathrm{Cu} \mathrm{K} \alpha 1$ radiation operated at $40 \mathrm{kV}$ and $30 \mathrm{~mA}$. Nitrogen adsorption/ desorption isotherms were conducted on a Quantachrome Autosorb $\mathrm{iQ}_{2}$, using the NLDFT method to evaluate surface area, pore volume and pore size distributions, from the adsorption branch of the isotherms. Diffuse reflectance (DR) UV-Vis data were obtained with an Agilent Technologies Cary 4000 UV-Vis Spectrophotometer with a Harrick Praying Mantis diffuse reflectance accessory. XPS spectra of samples 1-13 were recorded on a Thermo Scientific 
Table 1 Doehlert matrices 1 (DM1) and 2 (DM2)

\begin{tabular}{lllll}
\hline Sample & Factor 1 & Surfactant mass $/ \mathrm{g}$ & Factor 2 & Temperature $/{ }^{\circ} \mathrm{C}$ \\
\hline 1 & 0 & 7.9 & 0 & 80 \\
2 & 1 & 9.4 & 0 & 80 \\
3 & 0.5 & 8.6 & 0.866 & 97 \\
4 & -1 & 6.4 & 0 & 80 \\
5 & -0.5 & 7.2 & -0.866 & 63 \\
6 & 0.5 & 8.6 & -0.866 & 63 \\
7 & -0.5 & 7.2 & 0.866 & 97 \\
8 & 1 & 9.4 & 0 & 97 \\
9 & 0.5 & 9.0 & 0.866 & 104 \\
10 & -1 & 7.9 & 0 & 97 \\
11 & -0.5 & 8.3 & -0.866 & 90 \\
12 & 0.5 & 9.0 & -0.866 & 90 \\
13 & -0.5 & 8.3 & 0.866 & 104 \\
\hline
\end{tabular}

Change in surfactant mass and temperature

spectrometer with $\mathrm{Cu} \mathrm{K} \alpha$ radiation. The analyser was set at a pass energy of $20 \mathrm{eV}$ for high-resolution spectra of all the individual elements in each sample tested. Approximately $2-5 \mathrm{mg}$ of each powder sample was mounted on a stainless steel sample holder. The background was determined using the Shirleytype background correction, and the curves were fitted with Gaussian and Lorentzian product functions.

\section{Doehlert matrix approach to optimize synthesis}

The synthesis conditions were optimized by changing relevant parameters in the synthesis procedure, according to conditions specified by the Doehlert matrix model. The first matrix (Table 1 and Fig. 1, samples 1-7) was designed by changing surfactant mass and temperature. Thus, a two-factor system was designed, with seven total data point sets, consisting of one centre point and six other points lying at corners of a regular hexagon (refer ESI Sects. 1 and 2). In this approach, the coordinates of each point are values for factor 1 and factor 2; hence, two parameters are changed simultaneously for each experiment. The centre point consists of reference experimental conditions. Next, the parameters that would be factor 1 and factor 2 have to be decided, since they contain five (i.e. $0,1,-0.5,-1,0.5$ ) and three (i.e. 0, 0.866, $-0.866)$ values, respectively. In order to determine this, an understanding of which of these parameters would have a stronger impact on the final outcome of the study, in this case, catalytic performance is

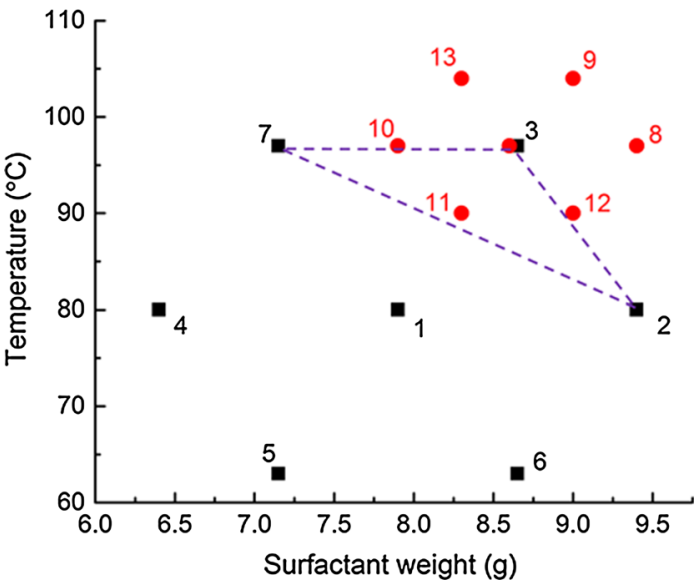

Figure 1 Samples 1-13, corresponding to Doehlert matrices 1 (DM1) and 2 (DM2). The triangle depicts the matrix region in DM1 that generated samples with high catalytic activity, which led to designing DM2.

necessary. The experience and prior knowledge of the researcher play a critical role here. Based on our understanding of factors that affect chemical and physical properties of the catalyst, we chose the surfactant mass as factor 1 , with five values. The parameter with less expected impact, i.e. homogenizing temperature, was chosen as factor 2, with three values. The centre point corresponded to $7.9 \mathrm{~g}$ of surfactant and $80{ }^{\circ} \mathrm{C}$ for the homogenizing temperature [39]. Exact values of factors 1 and 2 were calculated based on Eq. 1, given by the Doehlert model:

$P_{i}=x+a * F_{i}$ 
where $P_{i}=$ calculated value of parameter, $x=$ starting value of parameter, $a=$ probe limit of parameter, $F_{i}=$ value of factor (coded) for experiment $i, i=$ experiment number.

The experiments were later extended to design a second matrix (samples 8-13) with a narrower range of conditions. Limits for the first matrix were $\pm 1.5 \mathrm{~g}$ for surfactant mass and $\pm 17{ }^{\circ} \mathrm{C}$ for temperature. For the second matrix, limits were reduced to $\pm 0.8 \mathrm{~g}$ and $\pm 7{ }^{\circ} \mathrm{C}$, respectively. These parameters, their starting values and limits were chosen based on preliminary experiments, which indicated them to be the most influential features on the products formed [47]. A third matrix was designed by changing TEOS concentration and homogenizing temperature.

\section{Catalytic experiments}

MTSM samples were catalytically characterized via epoxidation of cyclohexene with TBHP according to a procedure adapted from the literature [65]. $25 \mathrm{mmol}$ of cyclohexene was mixed with $20 \mathrm{~mL}$ of decane as solvent in a batch reactor, followed by $100 \mathrm{mg}$ of MTSM catalyst. Next, approximately $7 \mathrm{~g}$ of $4 \AA$ molecular sieves were added to the reactor in order to remove moisture from the mixture. The mixture was kept under argon for $30 \mathrm{~min}$ to create an inert atmosphere and stirred at $60{ }^{\circ} \mathrm{C}$ for another $30 \mathrm{~min}$. $5.5 \mathrm{mmol}$ of $5.5 \mathrm{M}$ TBHP solution in decane was then added to initiate the reaction (ESI Scheme 1). Samples from the reaction mixture were withdrawn at relevant time periods and analysed by gas chromatograph using a GC-2014 Shimadzu Gas Chromatograph, employing a ZB-WAXplus Zebron capillary GC column.

Calibration curves for TBHP, cyclohexene and cyclohexene oxide were obtained using standard solutions. These were used for the determination of the relevant concentrations of the reaction mixtures, via GC analysis (ESI Figures S4, S5, S6 and S7).

\section{Kinetic studies}

Kinetic experiments were conducted on the most promising sample from the study, i.e. sample 7. This sample displayed the highest product yield and selectivity with excellent reagent conversion, during a 24-h reaction. Further epoxidation experiments were performed on this catalyst, and samples were taken out at time intervals $0,2,4,6,8$ and $24 \mathrm{~h}$, and immediately subjected to GC analysis.

\section{Results and discussion}

\section{Criteria behind synthesis optimization experiments}

The parameters of the MTSM microspheres were probed according to two routes: (1) change in morphology, i.e. particle size, shape and pore network properties, and (2) change in chemical structure, i.e. ratio of $\mathrm{Ti}$ to $\mathrm{Si}$. The former was achieved by changing the amount of surfactant added during templating, as well as the reaction temperature, and the latter by changing the amount of TEOS added during synthesis and the reaction temperature. Note that, in each case, two synthesis parameters were changed simultaneously. Two matrices (DM1 and DM2) were designed for route 1.

The first Doehlert matrix (DM1) was designed as a two-factor system, around surfactant mass and homogenizing temperature values known from the literature [39], as primary and secondary variables, respectively (Table 1, samples 1-7 and Fig. 1). Using the relevant multiplication factors given by the Doehlert model, six other synthetic conditions were calculated, and seven samples in total were synthesized and used as catalysts for the epoxidation of cyclohexene using TBHP as oxidant (Table 2, samples 1-7). Cyclohexene was in excess and TBHP was the limiting reagent with a molar ratio of 5:1.

Based on the results obtained in terms of the yield and selectivity of the desired product formed with respect to the limiting reagent (i.e. amount of epoxide formed with respect to TBHP), a region of high catalytic activity was identified. This included samples 2, 3 and 7, where sample 7 was the clearly superior catalyst (Fig. 1). Based on these, a second matrix (DM2) was designed to further investigate the region of high activity (samples 8-13, Fig. 1 and Table 2). The range of synthesis conditions for DM2 was narrowed down for optimal investigation of the relevant parameter region (Fig. 1). Although DM2 had reasonably high catalytic activity, the results of product yields and selectivity did not surpass those of DM1 sample 7; hence, sample 7 was considered as the optimal point and no further matrices were designed for route 1 . 
Table 2 Results of cyclohexene epoxidation with TBHP using MTSM as catalyst after 24-h reaction in batch

\begin{tabular}{|c|c|c|c|c|c|}
\hline $\begin{array}{l}\text { Doehlert matrix } \\
\text { number }\end{array}$ & Sample & $\begin{array}{l}\text { Cyclohexene conversion } \%^{\mathrm{a}} \\
( \pm 4)\end{array}$ & $\begin{array}{l}\text { TBHP conversion } \%{ }^{b} \\
( \pm 8)\end{array}$ & $\begin{array}{l}\text { Epoxide yield w.r.t TBHP } \\
\%^{\mathrm{c}}\end{array}$ & $\begin{array}{l}\text { Selectivity } \\
\%{ }^{\mathrm{d}}\end{array}$ \\
\hline \multirow[t]{7}{*}{ DM1 } & 1 & 7 & 83 & 56 & 65 \\
\hline & 2 & 12 & 94 & 53 & 55 \\
\hline & 3 & 17 & 97 & 66 & 68 \\
\hline & 4 & 5 & 69 & 26 & 35 \\
\hline & 5 & 4 & 65 & 10 & 15 \\
\hline & 6 & 8 & 79 & 30 & 38 \\
\hline & 7 & 12 & 95 & 77 & 81 \\
\hline \multirow[t]{6}{*}{ DM2 } & 8 & 8 & 96 & 47 & 49 \\
\hline & 9 & 17 & 96 & 49 & 51 \\
\hline & 10 & 15 & 95 & 54 & 57 \\
\hline & 11 & 10 & 97 & 49 & 50 \\
\hline & 12 & 13 & 93 & 48 & 52 \\
\hline & 13 & 11 & 97 & 54 & 56 \\
\hline
\end{tabular}

Refer to ESI, Sect. 6 for details on calculations for $a, b, c$ and d

Overall, sample 7 has much improved catalytic activity in terms of reagent conversion, product yield and selectivity compared to the original starting material, sample 1 . Sample 3 is the second-best catalyst, followed by sample 2, which gives similar yield and selectivity to sample 1 . Samples 4,5 and 6 can be identified as poor catalysts. This shows that utilization of the DM approach has been successful in significantly enhancing the catalytic activity of the MTSM material, using a relatively small number of experiments. Additionally, samples 8-13 exhibited similar catalytic activity with respect to each other. This means that the synthesis conditions could be altered within a certain range without compromising the product yields and selectivity of titanosilicates. Such findings are significant, especially in designing large-scale synthesis procedures, where high product formation is expected with minimum raw material and energy costs. Doehlert matrix studies could similarly guide the industrial-scale design of synthesis processes.

A second route to optimize the synthesis via changing the chemical structure was accomplished by means of a third Doehlert matrix, DM3 (ESI Table S4 and Figure S8). Here, TEOS concentration and homogenizing temperature were probed as primary and secondary changing parameters, respectively. The results of DM3 did not surpass those of DM1 in a significant manner. Therefore, no further experiments were conducted along this route.

\section{Characterization of MTSM}

Initial characterization of MTSM was performed with FTIR, Raman and DR-UV in order to measure the presence of the isolated $\mathrm{Ti}-\mathrm{O}-\mathrm{Si}$ species that are important in catalysis. In the FTIR spectra, the characteristic peak at $945 \mathrm{~cm}^{-1}$, corresponding to the Ti$\mathrm{O}-\mathrm{Si}$ asymmetric stretching mode, was observed $[69,70]$ (Fig. 2-top left). The large peak at $1062 \mathrm{~cm}^{-1}$ corresponds to the antisymmetric $\mathrm{Si}-\mathrm{O}-\mathrm{Si}$ stretching mode and the peak at $800 \mathrm{~cm}^{-1}$ corresponds to the $\mathrm{O}-\mathrm{Si}-\mathrm{OH}$ bending mode, as compared with $\mathrm{SiO}_{2}$ [69]. The Raman spectrum also shows the characteristic Ti-O-Ti bands at 955 and $1100 \mathrm{~cm}^{-1}$ for the symmetric and the antisymmetric stretch, respectively (Fig. 2-top right) [71, 72].

Characteristic $\mathrm{TiO}_{2}$ bands were observed for commercial anatase at 150, 395, 515 and $640 \mathrm{~cm}^{-1}$, whereas these were absent in the MTSM sample. DRUV clearly indicated the presence of a band at $218 \mathrm{~nm}$, confirming the presence of isolated $\mathrm{Ti}^{4+}$ in tetrahedral geometry [72] (Fig. 2-bottom left). However, a broad shoulder at $270 \mathrm{~nm}$ was also observed, indicating that there is considerable amount of bulk $\mathrm{TiO}_{2}$ in the material [73]. TGA was conducted to examine the stability of the synthesized MTSM and shows only one mass loss event between 30 to $140{ }^{\circ} \mathrm{C}$, resulting in a weight loss of approximately $6 \%$ (Fig. 2-bottom right). This could be attributed mainly to the loss of adsorbed water. A 

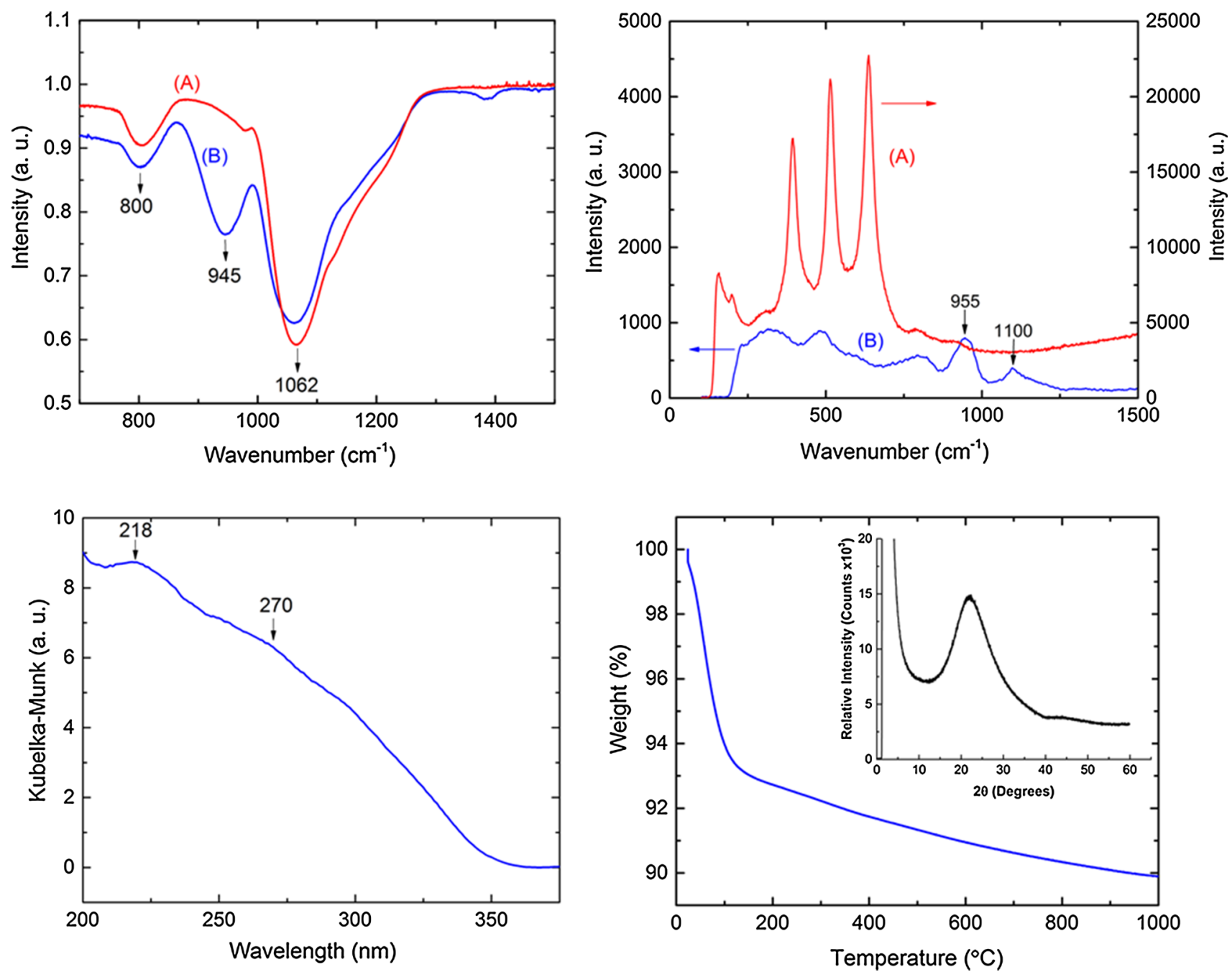

Figure 2 Characterization of MTSM sample 7. Top left-FTIR spectra of $\mathbf{a} \mathrm{SiO}_{2}$ and $\mathbf{b}$ MTSM showing characteristic Ti-O-Si peak at $945 \mathrm{~cm}^{-1}$, top right-Raman spectra of a Commercial anatase $\mathrm{TiO}_{2}$ and b MTSM, showing characteristic peaks at 955 and $1100 \mathrm{~cm}^{-1}$, bottom left-diffuse reflectance UV-Vis,

further gradual loss of mass was observed that continued until $1000{ }^{\circ} \mathrm{C}$, and could be due to loss of residual kerosene. The MTSM material appeared to be thermally stable and retained about $90 \%$ of its total weight, even at $1000{ }^{\circ} \mathrm{C}$.

Imaging techniques were used to investigate the morphology of the synthesized MTSM samples. SEM images revealed that the material was of microspherical morphology (Fig. 3). These were predominantly 20-30 $\mu \mathrm{m}$ in diameter and were mostly fused together. The microspheres appeared to be solid; however, some of them contained visible pores of 1-3 $\mu \mathrm{m}$, possibly from trapped surfactant and oil, which was removed during calcination. Note that

indicating the presence of isolated tetrahedral $\mathrm{Ti}$ at $218 \mathrm{~nm}$ and Ti-O-Ti oligomers at $270 \mathrm{~nm}$. Bottom right-TGA shows a mass loss at $30-140{ }^{\circ} \mathrm{C}$, indicating loss of water, inset - PXRD data showing one broad peak, indicating that the material is amorphous.

these spheres are not hollow as in the method described by Li and Coppens [39], due to minor changes in synthesis procedure; as the reactions are not diffusion limited in microspheres, a hollow interior serves no particular purpose for the epoxidation reaction. EDX elemental analysis revealed the particular purpose for the epoxidation reaction. EDX elemental analysis revealed that the material was only composed of $\mathrm{Si}, \mathrm{O}, \mathrm{Ti}$ and residual $\mathrm{C}$ (ESI Table S5, Figure S9). No other elements were present in any significant amount. Furthermore, HRTEM images of the crushed material indicated a predominantly amorphous morphology with small crystalline regions (ESI Figure S10). The amorphous 

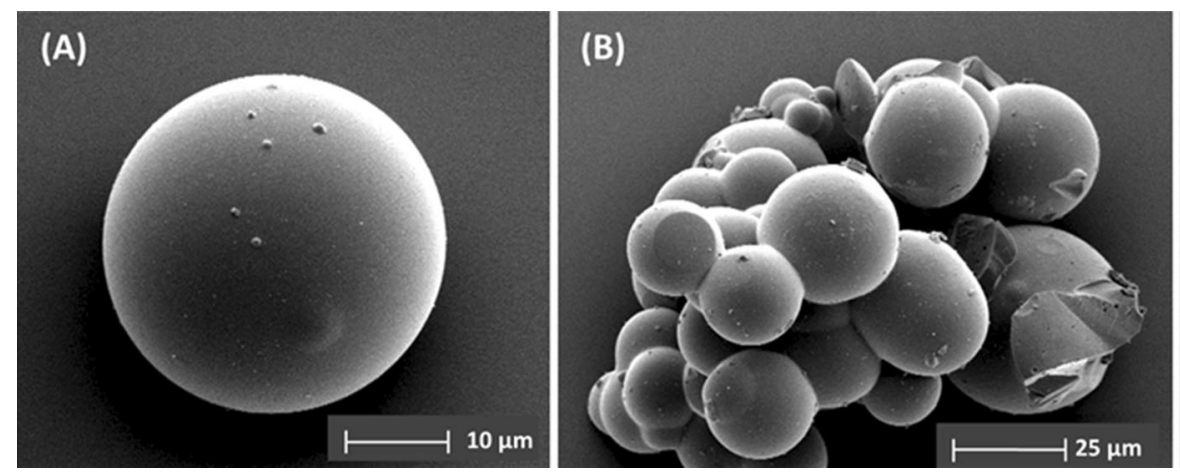

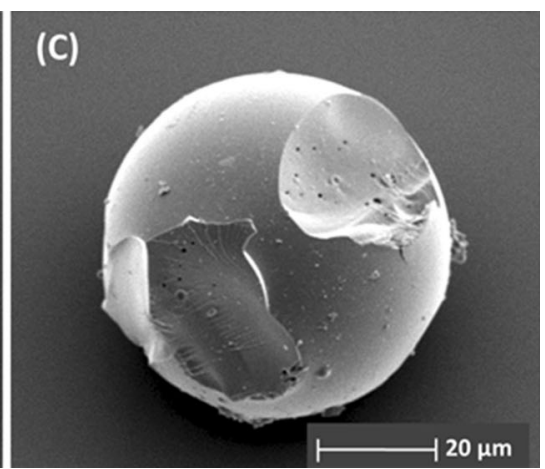

Figure 3 SEM images of MTSM microspheres, after calcination at $750{ }^{\circ} \mathrm{C}$ for $6 \mathrm{~h}$ : $\mathbf{a}$ an individual sphere; $\mathbf{b}$ cluster of spheres fused together, indicating average sizes of $20-30 \mu \mathrm{m}$; c a crushed

nature of the material was confirmed by PXRD experiments, where a single broad peak was observed with no indication of any sharp peaks (Fig. 2-bottom right-inset).

\section{Determining the factors that affect catalytic activity}

We found that changing the synthetic conditions systematically and simultaneously was an effective way to find conditions that generate significantly enhanced catalytic activity and selectivity of the MTSM material (Tables 1 and 2). A combination of higher temperatures and moderate surfactant amounts led to the synthesis of more effective catalysts. Sample 7, for example, gave 38 and 25\% higher product yield and selectivity, respectively, compared to the starting point (Table 2, sample 1 versus 7 ). However, high surfactant concentrations and temperatures became ineffective beyond certain limits. This was evidenced by the catalytic activity results of the matrix DM2 (Table 2, samples 8-13).

The two predominant factors that affected the catalytic activity of porous titanosilicates were the number of isolated $\mathrm{Ti}^{4+}$ sites per unit weight and the nature of the pore network [74]. Nitrogen physisorption (Fig. 4) and XPS measurements (Table 3 and Fig. 5) were conducted on samples 1-7, in order to investigate these factors thoroughly. BET analysis revealed a direct relationship between MTSM properties (i.e. surface area, pore size distribution, pore volume) and catalytic activity. The highest surface area, pore volume and mesopore size were associated with the best catalyst, sample 7 . The samples with high to moderate surface areas and microsphere, revealing that the material is mostly solid inside, with a few macropores from the surfactant oil emulsion, which was calcined.

pore volumes and pore sizes, such as 3, 2 and 1, had good to moderate catalytic activity, accordingly. Samples 5 and 6, which were clearly poor catalysts, had much smaller pore diameters, limiting access to the catalytic active sites by the bulky cyclohexene and TBHP reagents.

Furthermore, sample 6 had the smallest pore volume out of which $60 \%$ were micropores. Surface area and pore volume for sample 5 (which was the worst catalyst) could not be calculated as the isotherms did not close (ESI Figure S11). Sample 4, despite having a high surface area and pore volume, was a rather poor catalyst, possibly due to having a large micropore fraction $(16 \%)$, which hindered accessibility of reagents to the active sites.

XPS measurements were conducted on all samples of DM1 (samples 1-7), in order to investigate the effect of the amount of active tetrahedral $\mathrm{Ti}^{4+}$ per unit volume on their catalytic activity (Table 3 and ESI Table S2). The XPS Ti $2 p$ spectra were deconvoluted into Ti $2 \mathrm{p}_{3 / 2}$ and Ti $2 \mathrm{p}_{1 / 2}$ peaks at 459 and $465 \mathrm{eV}$, respectively, with constrained 2:1 intensity ratio $[75,76]$. The two peaks occur due to multiplet splitting from different spin distributions of the electrons of the Ti band structure $[77,78]$. The two Ti $2 p$ peaks indicated that $\mathrm{Ti}^{4+}$ was the only oxidation state of the MTSM material (Fig. 5a) and represent $\mathrm{Ti}^{4+}$ in octahedral (lower binding energy) and tetrahedral (higher binding energy) coordination [79-81]. The catalytically active, isolated $\mathrm{Ti}-\mathrm{O}-\mathrm{Si}$ sites were represented by the tetrahedral coordination ( $\mathrm{Ti} 2 \mathrm{p}_{1 / 2}$ ), whereas the octahedral coordination accounts for the inactive $\mathrm{Ti}-\mathrm{O}-\mathrm{Ti}$ oligomers and aggregated $\mathrm{TiO}_{2}$. Evidence of such $\mathrm{Ti}-\mathrm{O}-\mathrm{Ti}$ oligomers was found during diffuse reflectance UV-Vis experiments, with 

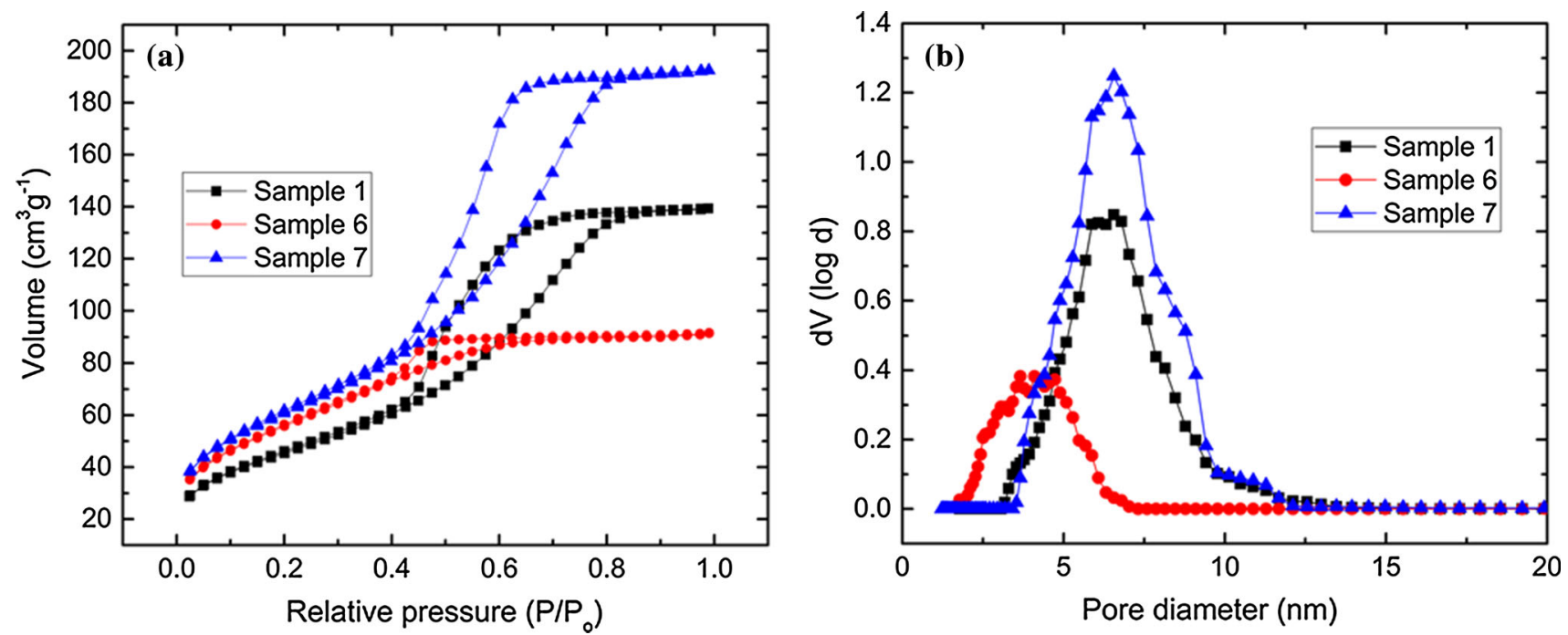

Figure 4 a Adsorption-desorption isotherms for samples 2, 3 and 7, using $\mathrm{N}_{2}$ at $77 \mathrm{~K}$. b Pore size distributions for samples 2, 3 and 7 , calculated using the NLDFT method.

Table 3 Nitrogen physisorption and XPS data for samples 1-7

\begin{tabular}{llllll}
\hline Sample & $\begin{array}{l}\text { BET surface area / } \\
\mathrm{m}^{2} \mathrm{~g}^{-1}\end{array}$ & $\begin{array}{l}\text { Total pore volume/ } \\
\mathrm{cm}^{3} \mathrm{~g}^{-1}\end{array}$ & $\begin{array}{l}\text { Micropore volume/ } \\
\%\end{array}$ & $\begin{array}{l}\text { Average pore diameter/ } \\
\mathrm{nm}\end{array}$ & $\begin{array}{l}\text { Tetrahedral Ti } 2 p_{1 / 2} / \\
\%\end{array}$ \\
\hline 1 & 166 & 0.208 & 6 & 6 & 0.74 \\
2 & 168 & 0.183 & 12 & 5 & 1.15 \\
3 & 142 & 0.165 & 6 & 6 & 0.7 \\
4 & 177 & 0.186 & 16 & 5.5 & 0.46 \\
5 & - & - & - & 2.5 & 1.43 \\
6 & 180 & 0.126 & 60 & 4 & 1.09 \\
7 & 231 & 0.292 & 4 & 9 & \\
\hline
\end{tabular}

the presence of a weak shoulder at $270 \mathrm{~nm}[82,83]$ (Fig. 2-bottom left).

The $\mathrm{O} 1 s$ spectra provided information about the different chemical environments of oxygen in the MTSM. However, the framework Ti species in the tetrahedral Ti-O-Si environment were impossible to identify versus the $\mathrm{Ti}-\mathrm{O}-\mathrm{Ti}$, as the former were masked by the Si-O-Si peak (Fig. $5 b$ ). The shoulder at $\sim 531 \mathrm{eV}$ generally corresponds to non-framework $\mathrm{TiO}_{2}$ species in octahedral environment [80, 81, 84]. The characteristic peak for $\mathrm{TiO}_{2}$, however, appeared at $530.1 \mathrm{eV}$, but the analogous peak of the synthesized MTSM was shifted by $\sim 1 \mathrm{eV}$ and appeared at $\sim 531 \mathrm{eV}$. Such deviation towards higher binding energies suggested the presence of tetrahedral Ti-OSi species $[79,80]$.

This was confirmed by the shift in binding energy for the Ti $2 p$ spectra $[80,84]$. The $\mathrm{Si}-\mathrm{O}-\mathrm{Si}$ peak of the silica framework appeared at $533 \mathrm{eV}$ and was analogous to that of $\mathrm{SiO}_{2}$ ( $\alpha$-quartz) [84].

For samples 1-7, it was evident that the samples with high catalytic activity such as 2, 3 and 7, contain higher amounts of tetrahedral $\mathrm{Ti}^{4+}$ (Table 3). However, the effect is not linear, as poor catalysts, such as sample 6, contain high amounts of tetrahedral $\mathrm{Ti}^{4+}$ active sites, but these are not accessible to the reagents, due to the presence of large amounts of micropores. Clearly, a combination of high surface area, pore volume, pore size and isolated $\mathrm{Ti}^{4+}$ amount is necessary to bring about increased catalytic activity. The Doehlert matrix approach allowed exploration of synthetic conditions that lead to such desirable properties, in a relatively short time frame.

Kinetic experiments were conducted for the best catalyst, sample 7 , in order to determine reaction order and rate coefficient. Samples were analysed at 

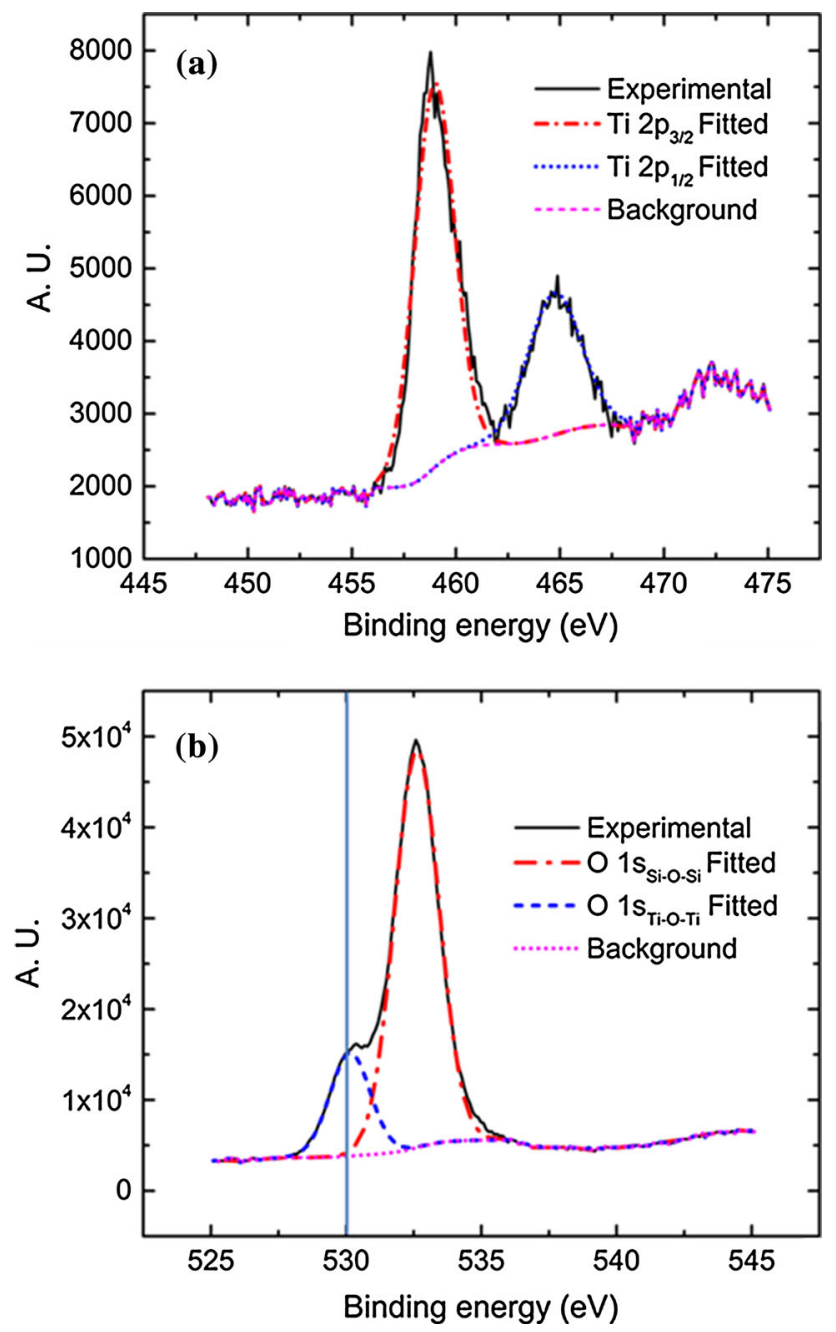

Figure 5 a XPS O $1 \mathrm{~s}$ spectra of sample 7, before and after reaction. b XPS O 1 s spectra for sample 7 . Vertical line indicates position of pure $\mathrm{TiO}_{2}(530 \mathrm{eV})$.

$0,2,4,6,8$ and $24 \mathrm{~h}$. A plot of $\ln [\mathrm{TBHP}]$ vs. time for the 2-24 h period appeared linear (Fig. 6a), indicating that the reaction follows, effectively, first-order kinetics after a short initiation time [85]. The conversion of the limiting reagent TBHP with time decayed exponentially during this period (Fig. 6b), also in accordance with first-order kinetics. However, for the 0-2 $\mathrm{h}$ time period, a more rapid TBHP conversion was observed. The effective rate constant, $k$, for 2-24 h can be determined from the slope of the linear drop of $\ln [\mathrm{TBHP}]$ vs. time and was found to be $0.11 \mathrm{~h}^{-1}$. Turnover frequency (TOF) values with respect to TBHP conversion were calculated for the reaction times of 2-24 h, based on the instantaneous reaction rates of TBHP and the concentration of $\mathrm{Ti}$ active sites (Eq. 2): [86, 87]
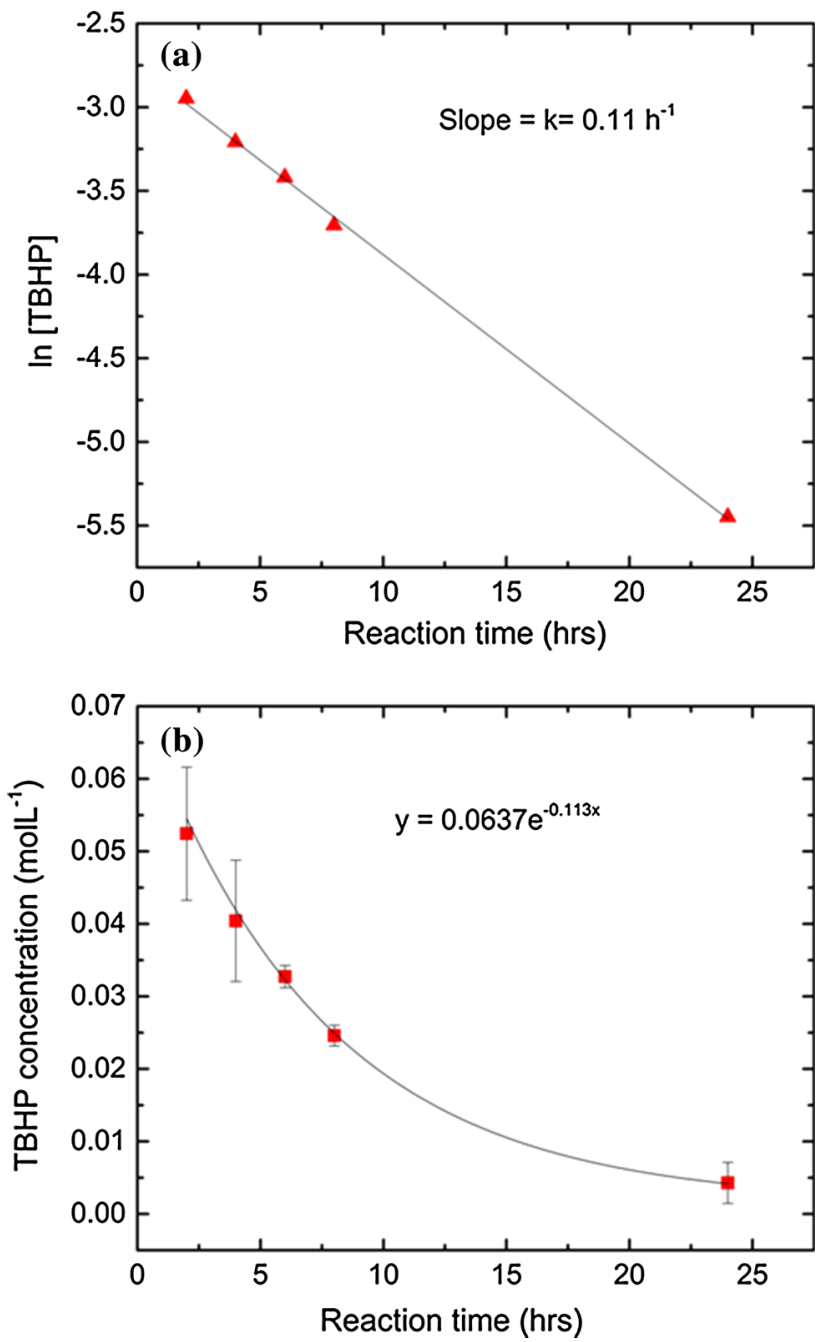

Fig. 6 Kinetic experiments for sample 7. a $\ln [\mathrm{TBHP}]$ vs reaction time. $\mathbf{b}$ TBHP concentration vs reaction time. Kinetics for, at least, 2-24 h are effectively first order.

$\mathrm{TOF}\left(h^{-1}\right)=\frac{\text { Instantaneous reaction rate }}{[\mathrm{Ti} \text { active sites }]}$

The relevant weight $\%$ of $\mathrm{Ti}$ was calculated from the atomic \% obtained from XPS, while the slope of the tangent line at each reaction time from Fig. $6 \mathrm{~b}$ was used to find the instantaneous reaction rate. TOF is represented according to two ways: using the amount of tetrahedral $\mathrm{Ti}$ active sites and the total amount of Ti (Table 4). The reason for calculating two types of TOF values is to distinguish the results between catalytically active $\mathrm{Ti} 2 p_{1 / 2}$ sites and the total amount of $\mathrm{Ti}$, which also includes $\mathrm{TiO}_{2}$, i.e. the inactive $\mathrm{Ti}$ $2 p_{2 / 3}$. The use of tetrahedral $\mathrm{Ti}$ is a better representation of the catalytic activity of the MTSM material, since DR-UV revealed the presence of significant 
Table 4 TOF values calculated using the tetrahedral Ti content of sample 7, with respect to TBHP conversion for different reaction times

\begin{tabular}{lcc}
\hline Reaction time/h & TOF based on tetrahedral Ti $/ \mathrm{h}^{-1}$ & TOF based on total Ti $/ \mathrm{h}^{-1}$ \\
\hline 2 & 156 & 52 \\
4 & 125 & 42 \\
6 & 99 & 33 \\
8 & 79 & 26 \\
24 & 13 & 4 \\
\hline
\end{tabular}

amounts of $\mathrm{TiO}_{2}$ (Fig. 2-bottom left). The TOF values indicate that the reaction is reasonably fast, in comparison with other non-functionalized titanosilicates, used under similar conditions, for epoxidation of cyclohexene with TBHP [88-91]. ICP-AES experiments were conducted on samples 1-7 to evaluate the bulk Ti content in the materials (ESI, Table S2). Unlike XPS, ICP reveals the weight \% of Ti. However, it does not reveal the oxidation or coordination states of different Ti species and therefore cannot distinguish between the amounts of active and non-active species. Therefore, tetrahedral Ti atomic \% given by XPS was chosen to normalize the data, as it best represents the active species of MTSM.

It is evident from the above discussion that using a statistically based modelling approach to guide the optimization of the synthesis conditions of titanosilicates brings about materials with superior catalytic activity, in a relatively easy way. It also reveals important structure-function relationships. Surfactant templating is shown to be an effective technique for generating mesoporous catalyst structures. The catalytic activity of the MTSM material is shown to be dependent upon this unique structure, along with its chemical composition. More detailed insights into the active site could be obtained in the future by operando studies, EXAFS and XANES measurements [92].

\section{Conclusions}

We have successfully demonstrated the implementation of the Doehlert matrix approach for optimizing the synthesis of titanosilicates that leads to improved catalytic activity, achieved via simultaneously changing two synthesis parameters, which are homogenizing temperature and surfactant weight. Extensive characterization of the MTSM materials revealed that a complex combination of factors affects the catalytic activity. These include physical parameters, such as pore size distribution and micro-mesoporosity, together with chemical parameters, such as the amount of framework $\mathrm{Ti}^{4+}$. Kinetic experiments revealed that the catalyst has first-order kinetics between 2-24 h. The sample synthesized by homogenizing at $97{ }^{\circ} \mathrm{C}$, with $7.2 \mathrm{~g}$ of surfactant, generated the best catalyst, with a high surface area and high accessible mesoporosity, and resulted in 95\% conversion of TBHP and $81 \%$ selectivity towards epoxide. Understanding such intricacies that relate chemical structure and morphology to catalytic activity provides critical information leading to further progress in the field of titanosilicate catalysis. The Doehlert matrix optimization method thus enables the discovery of new and effective catalysts by efficiently and simultaneously probing synthesis parameters.

\section{Supplementary information}

Electronic supplementary information is available free of charge at http://link.springer.com/. Advantages of using the Doehlert matrix model compared to selected other statistical models; Doehlert matrix experimental design; reaction scheme of cyclohexene with TBHP under experimental conditions; calibration curves for TBHP, cyclohexene and cyclohexene oxide; gas chromatogram of reaction mixture; details on calculations for catalytic experiments in Table 2 (calculation of reagent conversions, product yield, selectivity); XPS and ICP data; details of calculation of turnover frequencies; Doehlert matrix experiments for chemical structure modification: change in TEOS concentration and temperature; EDX experimental details, elemental analysis, image and spectrum; HRTEM imaging of catalyst; BET surface area of samples 2, 3, 4 and 5 .

\section{Acknowledgements}

The authors gratefully acknowledge the EPSRC for funding via a "Frontier Engineering" Award, EP/ 
K038656/1 and also acknowledge the valuable contributions from Prof Paul McMillan and Dr Steven Firth of the Department of Chemistry, UCL, for assistance with Raman spectroscopy, Prof Zheng X. Guo and Dr Kaipei Qiu of the Department of Chemistry, UCL, for BET measurements, Mr Jim Davy of the Department of Earth Sciences, UCL, and Dr Nicola Mordan of the Eastman Dental Institute, UCL, for SEM imaging, Dr Jeremy Cockcroft of the Department of Chemistry, UCL, for XRD measurements, Dr Guanghua Ye of the Department of Chemical Engineering, UCL (currently at East China University of Science and Technology), for TGA measurements, Dr Junwang Tang of the Department of Chemical Engineering, UCL, for providing us with a batch reactor, Mr Antonio Seccomandi, MEng, Chemical Engineering, UCL, for assistance with experiments and IFPEN, Lyon, France, for ICP-AES measurements.

\section{Compliance with ethical standards}

Conflict of interest The authors declare that they have no conflict of interest.

Electronic supplementary material: The online version of this article (https://doi.org/10.1007/ s10853-018-2057-2) contains supplementary material, which is available to authorized users.

Open Access This article is distributed under the terms of the Creative Commons Attribution 4.0 International License (http://creativecommons.org/ licenses/by/4.0/), which permits unrestricted use, distribution, and reproduction in any medium, provided you give appropriate credit to the original author(s) and the source, provide a link to the Creative Commons license, and indicate if changes were made.

\section{References}

[1] Perera AS, Coppens M-O (2016) Titano-silicates: highlights on development, evolution and application in oxidative catalysis. In: Spivey JJ (ed) Catalysis. RSC Publishing, pp 119-143

[2] Huybrechts DRC, De Bruycker L, Jacobs PA (1990) Oxyfunctionalization of alkanes with hydrogen peroxide on titanium silicalite. Nature 345:240-242
[3] Roffia P, Leofanti G, Cesana A et al (1990) Cyclohexanone ammoximation: a break through in the 6-caprolactam production process. Stud Surf Sci Catal 55:43-52

[4] Notari B (1993) Titanium silicalites. Catal Today 18:163-172

[5] Tatsumi T, Nakamura M, Negishi S, Tominaga H (1990) Shape-selective oxidation of alkanes with $\mathrm{H}_{2} \mathrm{O}_{2}$ catalysed by titanosilicate. J Chem Soc, Chem Commun 6:476-477

[6] Anderson MW, Terasaki O, Ohsuna T et al (1994) Structure of the microporous titanosilicate ETS-10. Nature 367:347-351

[7] Sato T, Dakka J, Sheldon RA (1994) Titanium-substituted zeolite beta(Ti-Al- $\beta$ )-catalysed epoxidation of oct-1-ene with tert-butyl hydroperoxide(TBHP). J Chem Soc, Chem Commun 16:1887-1888

[8] De Raffele G, Aloise A, De Luca P, Vuono D, Tagarelli A, Nagy JB (2016) Kinetic and thermodynamic effects during the adsorption of heavy metals on ETS-4 and ETS-10 microporous materials. J Porous Mater 23:389-400

[9] Bagshaw SA, Pouzet E, Pinnavaia TJ (1995) Templating of mesoporous molecular sieves by nonionic polyethylene oxide surfactants. Science 269:1242-1244

[10] Corma A, Navarro MT, Pariente JP (1994) Synthesis of an ultralarge pore titanium silicate isomorphous to MCM-41 and its application as a catalyst for selective oxidation of hydrocarbons. J Chem Soc, Chem Commun 2:147-148

[11] Morey MS, O'Brien S, Schwarz S, Stucky GD (2000) Hydrothermal and postsynthesis surface modification of cubic, MCM-48, and ultralarge pore SBA-15 mesoporous silica with Titanium. Chem Mater 12:898-911

[12] Tanev PT, Chibwe M, Pinnavaia TJ (1994) Titanium-containing mesoporous molecular sieves for catalytic oxidation of aromatic compounds. Nature 368:321-323

[13] Sayari A, Reddy KM, Moudrakovski IL (1995) Synthesis of $\mathrm{V}$ and Ti modified MCM-41 mesoporous molecular sieves. Stud Surf Sci Catal 98:19-21

[14] Ferdov S (2014) Layered titanosilicates for size- and patterncontrolled overgrowth of MFI zeolite. Cryst Eng Commun $16: 4467-4471$

[15] Ferdov S, Kostov-Kytin V, Petrov O (2002) A rapid method of synthesizing the layered titanosilicate JDF-L1. Chem Commun 16:1786-1787

[16] Du H, Fang M, Chen JC, Pang W (1996) Synthesis and characterization of a novel layered titanium silicate JDF-L1. J Mater Chem 6:1827-1830

[17] Roberts MA, Sankar G, Thomas JM et al (1996) Synthesis and structure of a layered titanosilicate catalyst with fivecoordinate titanium. Nature 381:401-404

[18] Millini R, Perego G, Parker WO Jr, Bellussi G, Carluccio L (1995) Layered structure of ERB-1 microporous borosilicate 
precursor and its intercalation properties towards polar molecules. Microporous Mater 4:221-230

[19] Yang B, Jiang J-G, Zhang K, Wu P (2016) Synthesis of novel titanosilicate catalysts by simultaneous isomorphous substitution and interlayer expansion of zeolitic layered silicates. Chem Mater 28:5295-5303

[20] Liu H, Yonezawa A, Kumagai K, Sano M, Miyake T (2015) Cs and Sr removal over highly effective adsorbents ETS-1 and ETS-2. J Mater Chem A 3:1562-1568

[21] Barreira LD, Lito PF, Antunes BM et al (2009) Effect of $\mathrm{pH}$ on Cadmium(II) removal from aqueous solution using titanosilicate ETS-4. Chem Eng J 155:728-735

[22] Noh YD, Komarneni S (2011) Mercury(II) exchange by highly charged swelling micas, sodium Engelhard titanosilicate-4, and sodium titanosilicate. Environ Sci Technol 45:6954-6960

[23] Oleksiienko O, Wolkersdorfer C, Sillanpää M (2017) Titanosilicates in cation adsorption and cation exchange-a review. Chem Eng J 317:570-585

[24] Figueiredo BR, Portugal I, Rocha J, Silva CM (2016) Fixedbed removal of $\mathrm{Cs}+$ from aqueous solutions by microporous silicate ETS-4: measurement and modeling of loading-regeneration cycles. Chem Eng J 301:276-284

[25] Mentzen BF, Gelin P (1995) The silicalite/p-xylene system : part I-flexibility of the MFI framework and sorption mechanism observed during $\mathrm{p}$-xylene pore-filling by X-ray powder diffraction at room temperature. Mater Res Bull 30:373-380

[26] Anson A, Lin CCH, Kuznicki SM, Sawada JA (2009) Adsorption of carbon dioxide, ethane, and methane on titanosilicate type molecular sieves. Chem Eng Sci 64:3683-3687

[27] Tiscornia I, Irusta S, Téllez C, Coronas J, Santamaría J (2008) Separation of propylene/propane mixtures by titanosilicate ETS-10 membranes prepared in one-step seeded hydrothermal synthesis. J Membr Sci 311:326-335

[28] Zecchina A, Areán CO, Palomino GT et al (1999) The vibrational spectroscopy of $\mathrm{H}_{2}, \mathrm{~N}_{2}, \mathrm{CO}$ and $\mathrm{NO}$ adsorbed on the titanosilicate molecular sieve ETS-10. Phys Chem Chem Phys 1:1649-1657

[29] Jeong H-K, Krohn J, Sujaoti K, Tsapatsis M (2002) Oriented molecular sieve membranes by heteroepitaxial growth. J Am Chem Soc 124:12966-12968

[30] Li X, Zhou C, Lin Z et al (2011) Titanosilicate AM-3 membrane: a new potential candidate for $\mathrm{H}_{2}$ separation. Microporous Mesoporous Mater 137:43-48

[31] Sebastián V, Téllez C, Coronas J, Santamaría J (2008) Formation of micro/macroporous hierarchical spheres of titanosilicate umbite. Eur J Inorg Chem 2008:2448-2453
[32] Lin Z, Rocha J, Navajas A, Téllez C, Coronas J, Santamaría J (2004) Synthesis and characterisation of titanosilicate ETS10 membranes. Microporous Mesoporous Mater 67:79-86

[33] Ferraris G (2006) Pillared materials from layer titanosilicates? Solid State Phenom 111:47-50

[34] Pérez-Carvajal J, Aranda P, Berenguer-Murcia A, CazorlaAmorós D, Coronas J, Ruiz-Hitzky E (2013) Nanoarchitectures based on layered titanosilicates supported on glass fibers: application to hydrogen storage. Langmuir 29:7449-7455

[35] Bhaumik A, Tatsumi T (1999) Intramolecular rearrangement of epoxides generated in situ over titanium silicate molecular sieves. J Catal 182:349-356

[36] Schofield LJ, Kerton OJ, McMora P, Bethell D, Ellwood S, Hutchings GJ (2002) Oxidation of -hydroxy containing monoterpenes using titanium silicate catalysts: comments on regioselectivity and the role of acidity. J Chem Soc Perkin Trans 2:1475-1481

[37] van der Waal JC, Rigutto MS, van Bekkum H (1998) Zeolite titanium beta as a selective catalyst in the epoxidation of bulky alkenes. Appl Catal A Gen 167:331-342

[38] Přech J (2018) Catalytic performance of advanced titanosilicate selective oxidation catalysts - a review. Catal Rev 60:71-131

[39] Li W, Coppens M-O (2005) Synthesis and characterization of stable hollow Ti-silica microspheres with a mesoporous shell. Chem Mater 17:2241

[40] Wu P, Xu H, Xu L, Liu Y, He M (2013) MWW-type titanosilicate: synthesis, structural modification and catalytic applications to green oxidations. Springer Briefs in Green Chemistry for Sustainability

[41] Chen Y, Huang Y, Xiu J, Han X, Bao X (2004) Direct synthesis, characterization and catalytic activity of titaniumsubstituted SBA-15 mesoporous molecular sieves. Appl Catal A Gen 273:185-191

[42] Wu P, Tatsumi T (2002) Postsynthesis, characterization, and catalytic properties in alkene epoxidation of hydrothermally stable mesoporous Ti-SBA-15. Chem Mater 14:1657-1664

[43] Corma A (1997) From microporous to mesoporous molecular sieve materials and their use in catalysis. Chem Rev 97:2373-2419

[44] Koyano KA, Tatsumi T (1997) Synthesis of titanium-containing MCM-41. Microporous Mater 10:259-271

[45] Ferreira SLC, dos Santos WNL, Bezerra MA, Lemos VA, Bosque-Sendra JM (2003) Use of factorial design and Doehlert matrix for multivariate optimisation of an on-line preconcentration system for lead determination by flame atomic absorption spectrometry. Anal Bioanal Chem $375: 443-449$ 
[46] Montgomery DC (1996) Design and analysis of experiments, 4th edn. John Wiley \& Sons Inc, New York

[47] Ferreira SLC, dos Santos WNL, Quintella CM, Neto BB, Bosque-Sendra JM (2004) Doehlert matrix: a chemometric tool for analytical chemistry—review. Talanta 63:1061-1067

[48] Box GEP, Wilson KB (1951) On the experimental attainment of optimum conditions. J R Stat Soc Ser B (Methodol) $13: 1-45$

[49] Box GEP, Behnken DW (1960) Some new three level designs for the study of quantitative variables. Technometrics 2:455-475

[50] Cavazzuti M (2013) Optimization methods: from theory to design scientific and technological aspects in mechanics. Springer-Verlag, Berlin Heidelberg

[51] Gong W, Cai Z, Jiang L (2008) Enhancing the performance of differential evolution using orthogonal design method. Appl Math Comput 206:56-69

[52] Doehlert DH (1970) Uniform shell designs. Appl Stat 19:231-239

[53] Bosque-Sendra JM, Nechar M, Rodriguez LC, Molina MFM (1995) Optimization of analytical methods by using Doehlert's designs. Anal Proc Incl Anal Commun 32:375-377

[54] Bezerra MA, Conceição ALB, Ferreira SLC (2004) Doehlert matrix for optimisation of procedure for determination of nickel in saline oil-refinery effluents by use of flame atomic absorption spectrometry after preconcentration by cloudpoint extraction. Anal Bioanal Chem 378:798-803

[55] Mendonça DR, Andrade HMC, Guimarães PRB et al (2011) Application of full factorial design and Doehlert matrix for the optimisation of beef tallow methanolysis via homogeneous catalysis. Fuel Process Technol 92:342-348

[56] Ferreira SLC, dos Santos HC, Fernandes MS, de Carvalho MS (2002) Application of Doehlert matrix and factorial designs in optimization of experimental variables associated with preconcentration and determination of molybdenum in sea-water by inductively coupled plasma optical emission spectrometry. J Anal At Spectrom 17:115-120

[57] Assis JCR, Teixeira JSR, Pontes LAM et al (2013) Using the Doehlert matrix as a tool for studying the influence of gasoline components on octane numbers. Fuel 113:744-749

[58] Hammami S, Ouejhani A, Bellakhal N, Dachraoui M (2009) Application of Doehlert matrix to determine the optimal conditions of electrochemical treatment of tannery effluents. J Hazard Mater 163:251-258

[59] Zhou B, Li Y, Gillespie J, He G-Q, Horsley R, Schwarz P (2007) Doehlert matrix design for optimization of the determination of bound deoxynivalenol in barley grain with trifluoroacetic acid (TFA). J Agric Food Chem 55:10141-10149

[60] Taramasso M, Perego G, Notari B (1983)
[61] Peregot G, Bellussi G, Corno C, Taramasso M, Buonomot F, Esposito A (1986) Titanium-silicalite: a novel derivative in the pentasil family. Stud Surf Sci Catal 28:129-136

[62] Harrison WTA, Gier TE, Stucky GD (1995) Single-crystal structure of $\mathrm{Cs} 3 \mathrm{HTi} 4 \mathrm{O} 4(\mathrm{SiO} 4) 3$ 4H2O, a titanosilicate pharmacosiderite analog. Zeolites 15:408-412

[63] Tatsumi T, Jappar N (1998) Properties of Ti-beta zeolites synthesized by dry-gel conversion and hydrothermal methods. J Phys Chem B 102:7126-7131

[64] Kubota Y, Koyama Y, Yamada T, Inagaki S, Tatsumi T (2008) Synthesis and catalytic performance of Ti-MCM-68 for effective oxidation reactions. Chem Commun 46:6224-6226

[65] Kosuge K, Singh PS (1999) Titanium-containing porous silica prepared by a modified sol-gel method. J Phys Chem B 103:3563-3569

[66] Rocha J, Anderson MW (2000) Microporous titanosilicates and other novel mixed octahedral-tetrahedral framework oxides. Eur J Inorg Chem 2000:801-818

[67] Catlow CRA, French SA, Sokol AA, Thomas JM (2005) Computational approaches to the determination of active site structures and reaction mechanisms in heterogeneous catalysts. Philos Trans R Soc A 363:913-936

[68] To J, Sherwood P, Sokol AA et al (2006) QM/MM modelling of the TS-1 catalyst using HPCx. J Mater Chem 16:1919-1926

[69] de Man AJM, Sauer J (1996) Coordination, structure, and vibrational spectra of titanium in silicates and zeolites in comparison with related molecules. An ab initio study. J Phys Chem 100:5025-5034

[70] Marchese L, Gianotti E, Dellarocca V et al (1999) Structurefunctionality relationships of grafted Ti-MCM41 silicas. Spectroscopic and catalytic studies. Phys Chem Chem Phys $1: 585-592$

[71] Knight DS, Pantano CG, White WB (1989) Raman spectra of gel-prepared titania-silica glasses. Mater Lett 8:156-160

[72] Ricchiardi G, Damin A, Bordiga S et al (2001) Vibrational structure of titanium silicate catalysts. A spectroscopic and theoretical study. J Am Chem Soc 123:11409-11419

[73] Yu J, Feng Z, Xu L et al (2001) Ti-MCM-41 Synthesized from colloidal silica and titanium trichloride: synthesis, characterization, and catalysis. Chem Mater 13:994-998

[74] Dal Santo V, Guidotti M, Psaro R, Marchese L, Carniato F, Bisio C (2012) Rational design of single-site heterogeneous catalysts: towards high chemo-, regio- and stereoselectivity. Proc R Soc A 468:1904-1926

[75] Biesinger MC, Lau LWM, Gerson AR, Smart RSC (2010) Resolving surface chemical states in XPS analysis of first row transition metals, oxides and hydroxides: $\mathrm{Sc}, \mathrm{Ti}, \mathrm{V}, \mathrm{Cu}$ and Zn. Appl Surf Sci 257:887-898 
[76] Wang A, Yu W, Fang Y et al (2015) Facile hydrothermal synthesis and optical limiting properties of $\mathrm{TiO} 2$-reduced graphene oxide nanocomposites. Carbon 89:130-141

[77] Briggs D, Seah MP (1990) Practical surface analysis: by auger and x-ray photoelectron spectroscopy. Wiley, Chichester

[78] Watts JF, Wolstenholme J (2005) An introduction to surface analysis by XPS and AES. Wiley-Interscience, Hoboken

[79] Contarini S, van der Heide PAW, Prakash AM, Kevan L (2002) Titaium coordination in microporous and mesoporous oxide materials by monochromated X-ray photoelectron spectroscopy and X-ray Auger electron spectroscopy. J Electron Spectrosc Relat Phenom 125:25-33

[80] Grohmann I, Pilz W, Walther C, Kosslick H, Tuan VA (1994) XPS-investigation of titanium modified MFI-type zeolites. Surf Interface Anal 22:403-406

[81] Moretti G, Salvi AM, Guascito MR, Langerame F (2004) An XPS study of microporous and mesoporous titanosilicates. Surf Interface Anal 36:1402-1412

[82] Baca M, Li WJ, Du P, Mul G, Moulijn JA, Coppens M-O (2006) Catalytic characterization of mesoporous Ti-Silica hollow spheres. Catal Lett 109:207-210

[83] Blasco T, Corma A, Navarro MT, Pariente JP (1995) Synthesis, characterization, and catalytic activity of Ti-MCM-41 structures. J Catal 156:65-74

[84] Wagner CD, Zatko DA, Raymond RH (1980) Use of the oxygen KLL Auger lines in identification of surface chemical states by electron spectroscopy for chemical analysis. Anal Chem 52:1445-1451
[85] Fogler HS (2015) Elements of chemical reaction engineering. Prentice Hall, Boston

[86] Davis ME, Davis RJ (2013) Fundamentals of chemical reaction engineering. Dover Publications, Newburyport

[87] Rogers TA, Bommarius AS (2010) Utilizing simple biochemical measurements to predict lifetime output of biocatalysts in continuous isothermal processes. Chem Eng Sci $65: 2118-2124$

[88] Chen LY, Chuah GK, Jaenicke S (1998) Ti-containing MCM-41catalysts for liquid phase oxidation of cyclohexene with aqueous $\mathrm{H}_{2} \mathrm{O}_{2}$ and tert-butyl hydroperoxide. Catal Lett 50:107-114

[89] Lin K, Pescarmona PP, Houthoofd K, Liang D, Van Tendeloo G, Jacobs PA (2009) Direct room-temperature synthesis of methyl-functionalized Ti-MCM-41 nanoparticles and their catalytic performance in epoxidation. J Catal 263:75-82

[90] Rogers MC, Adisa B, Bruce DA (2004) Synthesis and characterization of dendrimer-templated mesoporous oxidation catalysts. Catal Lett 98:29-36

[91] Xu L, Huang D-D, Li C-G et al (2015) Construction of unique six-coordinated titanium species with an organic amine ligand in titanosilicate and their unprecedented high efficiency for alkene epoxidation. Chem Commun 51:9010-9013

[92] Wachs IE (2005) Recent conceptual advances in the catalysis science of mixed metal oxide catalytic materials. Catal Today 100:79-94 